\title{
IJCARS_IPCAI 2019 special issue: conference information processing for computer-assisted interventions, 10th international conference 2019-part 1
}

\author{
Elvis C. S. Chen ${ }^{1} \cdot$ Kanako Harada $^{2} \cdot$ Raphael Sznitman $^{3}$
}

Published online: 23 May 2019

(c) CARS 2019

For its 10th edition, it is a great pleasure to present this special issue of IJCARS - international conference on information processing for computer-assisted interventions (IPCAI). IPCAI 2019 will be held at Le Couvent Des Jacobin, Rennes Convention Center, France, on June 18-19, 2019, in conjunction with the Computer Assisted Radiology and Surgery (CARS) Congress.

With a first edition in 2010, the IPCAI meeting has continued to provide a leading forum to discuss the latest developments in computer-assisted interventions (CAI). With a strong emphasis on research and practice, CAI focuses on computer-based tools and methodologies to support medical interventions with the overall objective to enable precise and safe systems for minimally invasive medical interventions. Hence, the domain is heavily anchored in bringing forward novel developments in enhanced planning, real-time imaging, instrument guidance and visualization, as well as situation awareness and cognition. To promote translational research, the IPCAI meetings seek to showcase papers presenting novel technical algorithms and theory, clinical

Raphael Sznitman

raphael.sznitman@artorg.unibe.ch

Elvis C. S. Chen

chene@robarts.ca

Kanako Harada

kanako@nml.t.u-tokyo.ac.jp

1 Imaging Research Laboratories, Robarts Research Institute, Western University, 1151 Richmond St., London, ON N6A 3K7, Canada

2 Mitsuishi-Suita Lab, Department of Bioengineering/Mechanical Engineering, School of Engineering, The University of Tokyo, Eng. Bldg 2-71C1, 7-3-1, Hongo, Bunkyo-ku, Tokyo 113-8656, Japan

3 ARTORG Research Center Biomedical Engineering, University of Bern, Murtenstrasse 50, 3008 Bern, Switzerland applications and hardware as well as software systems and their validation.

One differentiating aspect of IPCAI is that submitted manuscripts are expected to be of journal quality, with the goal to publish all accepted manuscripts in this special volume of the International Journal of Computer Assisted Radiology and Surgery (IJCARS). To this end, the IPCAI review process was rigorous and involved evaluation of each manuscript by two Area Chairs and at least three external reviewers. Authors were able to provide a revised version of their manuscript and a response to reviewers based on the reviewers' comments. The revised manuscripts were subject to an additional review process, ensuring that all reviewers' critiques were properly addressed, and that the quality was appropriate for journal publication. Prior to acceptance, manuscripts were discussed by the Program Chairs, Program Board members and the Conference chairs.

For this year's edition, IPCAI received 61 manuscript submissions, spanning a strong geographic representation from Europe, North America and Asia. In total, 35 manuscripts were accepted for presentation at IPCAI 2019. Accepted manuscripts reflected breadth of key topics in CAI and include: (i) Interventional imaging; (ii) Surgical planning and simulation; (iii) Tracking and navigation; (iv) Augmented reality and user interfaces; (v) Image processing and instrumentation for CAI; (vi) Interventional robotics; and (vii) System evaluation and validation.

As in previous years, the 2019 IPCAI meeting is designed to encourage interactions between attendees and allow time for constructive discussion. All authors of accepted manuscripts are invited to present their work in an initial short 5-min presentation. To this, each manuscript is also presented as a poster during a dedicated interactive session. The conference attendees will also be asked to vote for papers that they would like to see discussed further. The papers with the highest number of votes will be presented in extended 
format followed by questions from the attendees and the committee members.

As a novel aspect of the 2019 IPCAI edition, the CAI community was invited to submit long abstracts to showcase their work. Emphasis was given on presenting (i) exciting new breakthroughs relevant to IPCAI and (ii) novel ideas with preliminary but limited experimental validation. A total of 25 submissions were received, of which 11 were accepted for presentations at IPCAI 2019. These submissions were reviewed by the Program Chairs and General Chairs. Accepted long abstract submissions will be showcased by short 5-min oral presentations as well as an associated poster presentation.

We would like to take this opportunity to thank the Area Chairs, the Program Board and all of the reviewers for their dedication to maintaining the high quality of IPCAI and for selecting outstanding manuscripts that will no doubt stimulate exciting discussions at the meeting.

We acknowledge our generous sponsors for their continued support over the years that has enabled us to recognize both IPCAI authors and reviewers for their much-deserved dedication and scientific enthusiasm through several paper awards. The awards include the Outstanding Reviewer Award, Young Investigator Travel Award, Bench-to-Bedside Award and Best Paper Award, all made possible through their generous sponsorship. On behalf of all awardees, we are very grateful for this continued support!

We also thank all of the CARS Congress Organizers and Editorial Office for their continued help and support with logistics, as well as with the IJCARS manuscript management and review process.

Perhaps most importantly, we are grateful to all authors for choosing to submit their manuscripts to IPCAI and hope that the readers enjoy this special issue. The continued success of IPCAI demands our ongoing commitment.

\section{Elvis Chen, Kanako Harada and Raphael Sznitman} (Program Chairs)

Publisher's Note Springer Nature remains neutral with regard to jurisdictional claims in published maps and institutional affiliations. 